ANNALES

UNIVERSITATIS MARIAE CURIE-SKŁODOWSKA

LUBLIN - POLONIA

VOL. LXXII, 1

SECTIO AA

2017

\title{
Synthesis of diphenylphosphinic acid esters
}

\author{
Ewelina Korzeniowska \\ Department of Organic Chemistry, Marie Curie-Sklodowska University \\ Gliniana 33, 20-614 Lublin, Poland \\ e-mail: ewelina_korzeniowska@wp.pl
}

\begin{abstract}
A set of diphenylphosphinic acid esters can be prepared from chlorodiphenylphosphine and appropriate alcohol in the presence of trimethylamine, followed by addition of $\mathrm{H}_{2} \mathrm{O}_{2}$. These compounds are crucial substrates for nucleophilic substitution reaction which provides access to the many useful compounds in organophosphorus chemistry.
\end{abstract}

\section{INTRODUCTION}

The development of new methods for the synthesis of organophosphorus compounds is still an important part of organic chemistry due to the high demand for these compounds in organic synthesis as well as in asymmetric catalysis. Most of the methods for the synthesis of these compounds include the reactivity of the phosphorus atom, which depending on the structure might exhibit both electrophilic and nucleophilic properties. The phosphorus compounds having alkoxy groups bonded to the phosphorus atom are excellent substrates for the synthesis of various types of organophosphorus-compounds. This is due to the fact that the alkoxy groups are good leaving groups making them easier to exchange with other substituent.

In this context, the most important is the substitution reaction where phosphorus-oxygen bond is replaced by phosphorus-carbon bond because this provides phosphines and their derivatives, which have found wides 
use in organic chemistry, especially in organometallic catalysis. Imamoto and Tsuruda examined the effect of substituents on benzene ring on the reactivity of phosphinous acid-borane estres in nucleophilic substitution reaction [1]. The results indicate that reactivity of phosphinous acidborane estres depends largely on the structure of alkoxy substituent. Imamoto et all. explored a new method for the synthesis of the key intermediate, optical active tertiary phosphine-borane [2].

One of the steps in this synthesis was nucelophilic substitution of phosphinous acid-borane esters with organolithium compounds. Nucleophilic displacement reactions can proceed with inversion or retention of configuration at the phosphorus atom. Hoge submitted the diastereomeric mixture of phospholane-borane esters to the reaction with methyllithium and the appropriate tertiary phosphine-boranes were obtained with complete retention of stereochemistry [3]. Jugé and coworkers described an asymmetric synthesis of $P$-stereogenic 2-hydroxyaryl phosphine-borane [4]. This methodology was based on two steps: first, the stereoselective synthesis of chlorophosphine-borane from the corresponding oxazaphospholidine-borane which led to arylphosphonite-boranes derivatives and secondly, the intramolecular nucleophilic substitution affording the desired product without loss of enantiomeric purity. Oshiki and Imamoto explored the nucleophilic substitution reaction of optically pure methylphenylphosphinous acidborane methyl ester with organolithium reagents [5].

These results demonstrated that less sterically hindered reagents such as $m$-anisyllithium or $p$-anisyllithium react with substrate to afford the corresponding products with inversion of configuration, but $O$-substituted phenyllithium reagents lead to the substitution product with low enantiomeric excess. Nucleophilic substitution reaction is very important in the synthesis of phosphine ligands which have found many applications in asymmetric catalysis. Nettekoven at all. obtained two $C_{2}$-symmetrical $P$-chiral diphosphines BPAF and BPNF which were synthesized via stereocontrolled nucleophilic substitution reaction sequence [6]. The same group used the methodology described above for the synthesis of new ferrocene and biferrocenyl ligands [7, 8]. In recent years, significant studies have been undertaken in the asymmetric synthesis of tertiary mono- and diphosphine ligands which have found application in pharmaceutical, agrochemical industry and for production of aromatic fragrances [9]. In spite of the numerous examples of chiral phosphorus ligands described up to date, the search for more efficient methods of the synthesis of mono- and diphosphines with biaryl or metallocene bridge is 
still actually in phosphorus chemistry [10]. Jugé and co-workers described the asymmetric synthesis of mono- and diphosphines based on the nucleophilic substitution reaction of 1,1'-dilithioferrocene with two equivalents of oxazaphospholidine-borane [11]. Unfortunately, this approach led to the desired product with only $10 \%$ yield.

Others researchers obtained a set of phosphinous acid-borane amides as a results of nucleophilic substitution of alkoxy moiety in the oxazaphospholidine- borane with the carbon nucleophiles [12, 13]. Yang, Lugand and Mathieu demonstrated the possibility for an exchange of phosphorus-oxygen bond and phosphorus-nitrogen bond at the same time, but it requires additional two steps [14]. The first step in the synthesis of tertiary phosphine-boranes was the classic nucleophilic substitution of oxazaphospholidine-boranes leading to phosphinous acid-borane amides. Subsequent methanolysis of the intermediate affords the appropriate methyl esters which finally reacted with pyridine karbanion yielding the desired tertiary phosphine-borane. Stoop and Mezzetti conducted the synthesis of phosphine derivatives possessing bulky substituents in the same way $[15,16]$. Brown at all. synthesized the enantiomerically pure triaryl- and diarylvinyl-phosphine oxides by sequential nuclelophilic displacements which proceeded with retention of configuration at phosphorus atom. To summarize phosphinic esters are very important starting materials and intermediates in organophosphorus chemistry.

\section{EXPERIMENTAL SECTION}

\subsection{General}

All reactions were performed under an argon atmosphere. Only dry solvents were used and the glassware was heated under vacuum prior to use. Solvents for chromatography were distilled once before use and solvents for extraction were used as received. Diethyl ether was dried over sodium/benzophenone ketyl. Na, EtOH, $i-\mathrm{PrOH}, n-\mathrm{BuOH}, i-\mathrm{BuOH}$, $s$ - $\mathrm{BuOH}, c-\mathrm{HexOH}, \mathrm{H}_{2} \mathrm{O}_{2}$ and chlorodiphenylphosphine were comercially available and used as received.

\subsubsection{Analytics and Instruments}

The NMR spectras were recorded on $500 \mathrm{MHz}$ spectrometer in $\mathrm{CDCl}_{3}$ as a solvent at room temperature unless otherwise noted. Chemical shifts $(\delta)$ are reported in ppm relative to residual solvent peak. Mass 
spectra were recorded in electron ionization (EI) mode and GC was recorded using following parameters: pressure $97.9 \mathrm{kPa}$, total flow 19.5 $\mathrm{cm}^{3} / \mathrm{min}$, column flow $1.5 \mathrm{~cm}^{3} / \mathrm{min}$, linear velocity $44.9 \mathrm{~cm} / \mathrm{s}$, split 10 , temperature program $\left(70^{\circ} \mathrm{C}\right.$ hold $3 \mathrm{~min}, 70-340^{\circ} \mathrm{C} / 12^{\circ} \mathrm{C} / \mathrm{min}$ hold 9.5 min, total $35 \mathrm{~min}$ ) or pressure $65 \mathrm{kPa}$, total flow $23.9 \mathrm{~mL} / \mathrm{min}$, column flow $1.2 \mathrm{~cm}^{3} / \mathrm{min}$, linear velocity $36.8 \mathrm{~cm} / \mathrm{s}$, split 20 , temperature program $\left(80^{\circ} \mathrm{C}\right.$ hold $3 \mathrm{~min}, 80-250^{\circ} \mathrm{C} / 20^{\circ} \mathrm{C} / \mathrm{min}$ hold $5 \mathrm{~min}, 250-300^{\circ} \mathrm{C} / 10^{\circ} \mathrm{C} / \mathrm{min}$ hold $30.5 \mathrm{~min}$, total $50 \mathrm{~min}$ ). Thin layer chromatography (TLC) was performed with precoated silica gel plates and visualized by UV light, $\mathrm{KMnO}_{4}$ solution or iodide on silica gel. The reaction mixtures were purified by column chromatography over silica gel (60-240 mesh). Melting points were determined in a capillary tube and were uncorrected.

\subsection{General procedure for the synthesis of diphenylphosphinic acid esters}

$\mathrm{Ph}_{2} \mathrm{PCl}\left(5 \mathrm{~cm}^{3}, 0.028 \mathrm{~mol}\right)$ in diethyl ether $\left(60 \mathrm{~cm}^{3}\right)$ was placed in a flame-dried three-necked flask $\left(250 \mathrm{~cm}^{3}\right)$ containing magnetic stirrer and fitted with an inert gas inlet. The mixture was placed in cold water bath, and a mixture of alcohol $(0.028 \mathrm{~mol})$ and triethylamine $\left(3,9 \mathrm{~cm}^{3}\right.$, $0.028 \mathrm{~mol}$ ) was added dropwise. After the addition was complete, the mixture was left under argon at room temperature for two hours. It was then filtered with careful extrusion of oxygen and moisture. Then, the filtrate was re-cooled to $0^{\circ} \mathrm{C}$ and $30 \% \quad \mathrm{H}_{2} \mathrm{O}_{2}\left(20 \mathrm{~cm}^{3}\right)$ was added dropwise. The mixture was left under argon overnight. The reaction was quenched by addition of saturated $\mathrm{NH}_{4} \mathrm{Cl}$ solution $\left(5 \mathrm{~cm}^{3}\right)$ and extracted with $\mathrm{CHCl}_{3}\left(3 \times 10 \mathrm{~cm}^{3}\right)$. The combined organic phases were dried over $\mathrm{Na}_{2} \mathrm{SO}_{4}$, filtered, and evaporated under reduced pressure. The residue was purified by column chromatography using $\mathrm{CHCl}_{3} / \mathrm{MeOH}$.

\subsubsection{Diphenylphosphinic acid ethyl ester (2a)}

This compound was prepared according to the General Procedure from $\mathrm{Ph}_{2} \mathrm{PCl}\left(5 \mathrm{~cm}^{3}, 0.028 \mathrm{~mol}\right)$, EtOH $\left(1.63 \mathrm{~cm}^{3}, 0.028 \mathrm{~mol}^{2}, \mathrm{NEt}_{3}\right.$ $\left(3.9 \mathrm{~cm}^{3}, 0.028 \mathrm{~mol}\right)$ as a colorless oil; yield $1.415 \mathrm{~g}(21 \%) . R_{f}=0.61$ $\left(\mathrm{CHCl}_{3}: \mathrm{MeOH} 40: 1\right) ;{ }^{1} \mathrm{H} \mathrm{NMR}\left(500 \mathrm{MHz}, \mathrm{CDCl}_{3}\right): \delta=1.37\left(\mathrm{t}, J_{\mathrm{H}-\mathrm{H}}=\right.$ 7.1 Hz, 3H); 4.08-4.14 (m, 2H); 7.42-7.47 (m, 4H); 7.49-7.54 (m, 2H); 7.80-7.85 (m, 4H); ${ }^{13} \mathrm{C}$ NMR $\left(126 \mathrm{MHz}, \mathrm{CDCl}_{3}\right): \delta=16.45\left(\mathrm{~d}, J_{\mathrm{P}-\mathrm{C}}=6.4\right.$ $\mathrm{Hz}) ; 61.06\left(\mathrm{~d}, J_{\mathrm{P}-\mathrm{C}}=6.5 \mathrm{~Hz}\right) ; 128.46\left(\mathrm{~d}, J_{\mathrm{P}-\mathrm{C}}=13.6 \mathrm{~Hz}\right) ; 131.57\left(\mathrm{~d}, J_{\mathrm{P}-\mathrm{C}}=\right.$ $10.0 \mathrm{~Hz}) ; 131.65\left(\mathrm{~d}, J_{\mathrm{P}-\mathrm{C}}=137.1 \mathrm{~Hz}\right) ; 132.02\left(\mathrm{~d}, J_{\mathrm{P}-\mathrm{C}}=2.7 \mathrm{~Hz}\right) ;{ }^{31} \mathrm{P}$ NMR $\left(202 \mathrm{MHz}, \mathrm{CDCl}_{3}\right.$ ): $\delta=31,30$; GC: $t_{\mathrm{R}}=9.14 \mathrm{~min}$; GC-MS (EI, $70 \mathrm{eV}$ ), 
m/z (\%): ( $\left.\mathrm{M}^{+}\right) 246$ (7): 245 (10); 218 (29); 217 (100); 202 (27); 201 (26); 199 (37); 155 (28); 154 (13); 153 (11); 152 (19); 141 (44); 125 (19); 124 (10); 105 (23); 104 (99); 94 (12). Anal. Calcd for $\mathrm{C}_{14} \mathrm{H}_{15} \mathrm{O}_{2} \mathrm{P}: \mathrm{C} 68.29, \mathrm{H}$ 6.14. Found: C 67.40, H 5.25.

\subsubsection{Diphenylphosphinic acid iso-propyl ester (2b)}

This compound was prepared according to the General Procedure from $\mathrm{Ph}_{2} \mathrm{PCl}\left(5 \mathrm{~cm}^{3}, 0.028 \mathrm{~mol}\right), i$-PrOH $\left(2.16 \mathrm{~cm}^{3}, 0.028 \mathrm{~mol}^{2}, \mathrm{NEt}_{3}\right.$ $\left(3.9 \mathrm{~cm}^{3}, 0.028 \mathrm{~mol}\right)$ as a white solid, melting point: $93-95^{\circ} \mathrm{C}$; yield $5.384 \mathrm{~g}(74 \%) . R_{f}=0.79\left(\mathrm{CHCl}_{3}: \mathrm{MeOH} 30: 1\right) ;{ }^{1} \mathrm{H}$ NMR $(500 \mathrm{MHz}$, $\left.\mathrm{CDCl}_{3}\right): \delta=1.35\left(\mathrm{~d}, J_{\mathrm{H}-\mathrm{H}}=6.3 \mathrm{~Hz}, 6 \mathrm{H}\right) ; 4.62-4.71(\mathrm{~m}, 1 \mathrm{H}) ; 7.40-7.45(\mathrm{~m}$, $4 \mathrm{H}) ; 4.47-4.52(\mathrm{~m}, 2 \mathrm{H}) ; 7.79-7.84(\mathrm{~m}, 4 \mathrm{H}) ;{ }^{13} \mathrm{C} \mathrm{NMR}(126 \mathrm{MHz}$, $\left.\mathrm{CDCl}_{3}\right): \delta=24.26\left(\mathrm{~d}, J_{\mathrm{P}-\mathrm{C}}=4.6 \mathrm{~Hz}\right) ; 70.15\left(\mathrm{~d}, J_{\mathrm{P}-\mathrm{C}}=6.0 \mathrm{~Hz}\right) ; 128.34$ $\left(\mathrm{d}, J_{\mathrm{P}-\mathrm{C}}=12.7 \mathrm{~Hz}\right) ; 131.56\left(\mathrm{~d}, J_{\mathrm{P}-\mathrm{C}}=10.7 \mathrm{~Hz}\right) ; 131.86\left(\mathrm{~d}, J_{\mathrm{P}-\mathrm{C}}=2.7 \mathrm{~Hz}\right)$; $132.37\left(\mathrm{~d}, J_{\mathrm{P}-\mathrm{C}}=137.1 \mathrm{~Hz}\right) ;{ }^{31} \mathrm{P}$ NMR $\left(202 \mathrm{MHz}, \mathrm{CDCl}_{3}\right): \delta=29.77$; GC: $t_{\mathrm{R}}=9.05 \mathrm{~min} ; \mathrm{GC}-\mathrm{MS}(\mathrm{EI}, 70 \mathrm{eV}), \mathrm{m} / \mathrm{z}(\%):\left(\mathrm{M}^{+}\right)(2) ; 220(13) ; 219$ (100); 217 (38); 202 (21); 201 (42); 199 (24); 155 (11); 152 (13); 141 (35); 94 (10). Anal. Calcd for $\mathrm{C}_{15} \mathrm{H}_{17} \mathrm{O}_{2} \mathrm{P}: \mathrm{C}$ 69.22, H 6.58. Found: C 69.04, H 6.64 .

\subsubsection{Diphenylphosphinic acid butyl ester (2c)}

This compound was prepared according to the General Procedure from $\mathrm{Ph}_{2} \mathrm{PCl}\left(5 \mathrm{~cm}^{3}, 0.028 \mathrm{~mol}\right), n-\mathrm{BuOH}\left(2.57 \mathrm{~cm}^{3}, 0.028 \mathrm{~mol}^{2}, \mathrm{NEt}_{3}\right.$ $\left(3.9 \mathrm{~cm}^{3}, 0.028 \mathrm{~mol}\right)$ as a white solid, melting point: $83-87^{\circ} \mathrm{C}$; yield $3.672 \mathrm{~g}(74 \%) . R_{f}=0.46\left(\mathrm{CHCl}_{3}: \mathrm{MeOH} 40: 1\right) ;{ }^{1} \mathrm{H}$ NMR $(500 \mathrm{MHz}$, $\left.\mathrm{CDCl}_{3}\right): \delta=0.92\left(\mathrm{t}, J_{\mathrm{H}-\mathrm{H}}=7.41 \mathrm{~Hz}, 3 \mathrm{H}\right) ; 1.40-1.49(\mathrm{~m}, 2 \mathrm{H}) ; 1.68-1.74(\mathrm{~m}$, $2 \mathrm{H})$; 4.01-4.06 (m, 2H); 7.43-7.47 (m, 4H); 7.50-7.54 (m, 2H); 7.79-.84 (m, 4H); $\left.{ }^{13} \mathrm{C} \mathrm{NMR} \mathrm{(126} \mathrm{MHz,} \mathrm{CDCl}_{3}\right): \delta=13.60 ; 18.84 ; 32.55$ $\left(\mathrm{d}, J_{\mathrm{P}-\mathrm{C}}=7,3 \mathrm{~Hz}\right) ; 64.66\left(\mathrm{~d}, J_{\mathrm{P}-\mathrm{C}}=6.4 \mathrm{~Hz}\right) ; 128.45\left(\mathrm{~d}, J_{\mathrm{P}-\mathrm{C}}=12.7 \mathrm{~Hz}\right)$; $131.59\left(\mathrm{~d}, J_{\mathrm{P}-\mathrm{C}}=10.0 \mathrm{~Hz}\right) ; 131.66\left(\mathrm{~d}, J_{\mathrm{P}-\mathrm{C}}=137.2 \mathrm{~Hz}\right) ; 132.00\left(\mathrm{~d}, J_{\mathrm{P}-\mathrm{C}}=\right.$ $2.7 \mathrm{~Hz}) ;{ }^{31} \mathrm{P} \mathrm{NMR}\left(202 \mathrm{MHz}, \mathrm{CDCl}_{3}\right): \delta=31.11$; GC: $t_{\mathrm{R}}=9.78 \mathrm{~min}$; GCMS (EI, $70 \mathrm{eV}), \mathrm{m} / \mathrm{z}(\%):\left(\mathrm{M}^{+}\right) 274$ (0.5); 220 (14); 219 (100); 217 (25); 202 (11); 201 (40); 199 (17); 152 (10); 143 (20); 141 (46); 132 (47). Anal. Calcd for $\mathrm{C}_{16} \mathrm{H}_{19} \mathrm{O}_{2} \mathrm{P}: \mathrm{C}$ 60.06, H 6.98. Found: C 60.24, H 7.04.

\subsubsection{Diphenylphosphinic acid iso-butyl ester (2d)}

This compound was prepared according to the General Procedure from $\mathrm{Ph}_{2} \mathrm{PCl}\left(5 \mathrm{~cm}^{3}, 0.028 \mathrm{~mol}\right), i$-BuOH $\left(2.59 \mathrm{~cm}^{3}, 0.028 \mathrm{~mol}^{2}, \mathrm{NEt}_{3}\right.$ $\left(3.9 \mathrm{~cm}^{3}, 0.028 \mathrm{~mol}\right)$ as a white waxy solid, yield $3.742 \mathrm{~g}(74 \%) . R_{f}=0.52$ 
$\left(\mathrm{CHCl}_{3}: \mathrm{MeOH} 40: 1\right) ;{ }^{1} \mathrm{H}$ NMR $\left(500 \mathrm{MHz}, \mathrm{CDCl}_{3}\right): \delta=0.98\left(\mathrm{~d}, J_{\mathrm{H}-\mathrm{H}}=\right.$ $6.79 \mathrm{~Hz}, 3 \mathrm{H}) ; 1.51\left(\mathrm{~d}, J_{\mathrm{H}-\mathrm{H}}=18.25 \mathrm{~Hz}, 3 \mathrm{H}\right) ; 1.98-2.07(\mathrm{~m}, 1 \mathrm{H}) ; 3.80(\mathrm{t}$, $\left.J_{\mathrm{H}-\mathrm{H}}=6.4 \mathrm{~Hz}, 2 \mathrm{H}\right) ; 7.44-7.49(\mathrm{~m}, 4 \mathrm{H}) ; 7.51-7.56(\mathrm{~m}, 2 \mathrm{H}) ; 7.79-7.84(\mathrm{~m}$, $4 \mathrm{H}) ;{ }^{13} \mathrm{C}$ NMR $\left(126 \mathrm{MHz}, \mathrm{CDCl}_{3}\right): \delta=18.81 ; 20.62 ; 29.24\left(\mathrm{~d}, J_{\mathrm{P}-\mathrm{C}}=7,3\right.$ $\mathrm{Hz}) ; 70.95\left(\mathrm{~d}, J_{\mathrm{P}-\mathrm{C}}=6.6 \mathrm{~Hz}\right) ; 128.55\left(\mathrm{~d}, J_{\mathrm{P}-\mathrm{C}}=12.9 \mathrm{~Hz}\right) ; 131.58\left(\mathrm{~d}, J_{\mathrm{P}-\mathrm{C}}=\right.$ $10.3 \mathrm{~Hz}) ; 131.13\left(\mathrm{~d}, J_{\mathrm{P}-\mathrm{C}}=138.4 \mathrm{~Hz}\right) ; 132.21\left(\mathrm{~d}, J_{\mathrm{P}-\mathrm{C}}=3.1 \mathrm{~Hz}\right) ;{ }^{31} \mathrm{P}$ NMR $\left(202 \mathrm{MHz}, \mathrm{CDCl}_{3}\right): \delta=32.29$; GC: $t_{\mathrm{R}}=9.53 \mathrm{~min}$; GC-MS (EI, $70 \mathrm{eV}$ ),

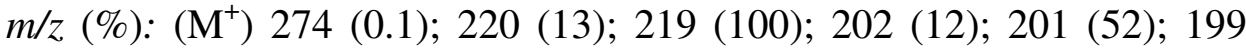
(10); 141 (41); 132 (46). Anal. Calcd for $\mathrm{C}_{16} \mathrm{H}_{19} \mathrm{O}_{2} \mathrm{P}: \mathrm{C} 70.06, \mathrm{H} 6.98$. Found: C 70.34, H 7.14.

\subsubsection{Diphenylphosphinic acid sec-butyl ester (2e)}

This compound was prepared according to the General Procedure from $\mathrm{Ph}_{2} \mathrm{PCl}\left(5 \mathrm{~cm}^{3}, 0.028 \mathrm{~mol}\right), s-\mathrm{BuOH}\left(2.57 \mathrm{~cm}^{3}, 0.028 \mathrm{~mol}^{3}\right) \mathrm{NEt}_{3}$ $\left(3.9 \mathrm{~cm}^{3}, 0.028 \mathrm{~mol}\right)$ as a white solid, melting point: $68-72^{\circ} \mathrm{C}$, yield $5.660 \mathrm{~g}(49 \%) . R_{f}=0.52\left(\mathrm{CHCl}_{3}: \mathrm{MeOH} 40: 1\right) ;{ }^{1} \mathrm{H}$ NMR $(500 \mathrm{MHz}$, $\left.\mathrm{CDCl}_{3}\right): \delta=0.91\left(\mathrm{t}, J_{\mathrm{H}-\mathrm{H}}=7.42 \mathrm{~Hz}, 3 \mathrm{H}\right) ; 1.31\left(\mathrm{~d}, J_{\mathrm{H}-\mathrm{H}}=6.31 \mathrm{~Hz}, 3 \mathrm{H}\right)$; 1.60-1.77 (m, 2H); 4.46-4.54 (m, 1H); 7.41-7.46 (m, 4H); 7.48-7.53 (m, $2 \mathrm{H}) ; 7.79-7.85(\mathrm{~m}, 4 \mathrm{H}) ;{ }^{13} \mathrm{C} \mathrm{NMR}\left(126 \mathrm{MHz}, \mathrm{CDCl}_{3}\right): \delta=9.38 ; 21.41(\mathrm{~d}$, $\left.J_{\mathrm{P}-\mathrm{C}}=3.1 \mathrm{~Hz}\right) ; 30.68\left(\mathrm{~d}, J_{\mathrm{P}-\mathrm{C}}=5.0 \mathrm{~Hz}\right) ; 74.69\left(\mathrm{~d}, J_{\mathrm{P}-\mathrm{C}}=6.4 \mathrm{~Hz}\right) ; 128.31$ $\left(\mathrm{d}, J_{\mathrm{P}-\mathrm{C}}=12.3 \mathrm{~Hz}\right) ; 131.55\left(\mathrm{~d}, J_{\mathrm{P}-\mathrm{C}}=10.0 \mathrm{~Hz}\right) ; 131.64\left(\mathrm{~d}, J_{\mathrm{P}-\mathrm{C}}=10.0 \mathrm{~Hz}\right)$; $131.83\left(\mathrm{~d}, J_{\mathrm{P}-\mathrm{C}}=2.7 \mathrm{~Hz}\right) ; 131.85\left(\mathrm{~d}, J_{\mathrm{P}-\mathrm{C}}=2.7 \mathrm{~Hz}\right) ; 132.44\left(\mathrm{~d}, J_{\mathrm{P}-\mathrm{C}}=\right.$ $137.2 \mathrm{~Hz}) ; 132.61\left(\mathrm{~d}, J_{\mathrm{P}-\mathrm{C}}=137.2 \mathrm{~Hz}\right) ;{ }^{31} \mathrm{P}$ NMR $\left(202 \mathrm{MHz}, \mathrm{CDCl}_{3}\right): \delta=$ 29.57; GC: $t_{\mathrm{R}}=9.12 \mathrm{~min}$; GC-MS (EI, $\left.70 \mathrm{eV}\right), \mathrm{m} / z(\%):\left(\mathrm{M}^{+}\right) 274(0.5)$; 220 (14); 219 (100); 217 (10); 202 (16); 201 (70); 199 (14); 152 (10); 141 (40); 132 (39). Anal. Calcd for $\mathrm{C}_{16} \mathrm{H}_{19} \mathrm{O}_{2} \mathrm{P}: \mathrm{C}$ 70.06, H 6.98. Found: C 69.96, H 7.15.

\subsubsection{Diphenylphosphinic acid cyclohexyl ester (2f)}

This compound was prepared according to the General Procedure from $\mathrm{Ph}_{2} \mathrm{PCl}\left(5 \mathrm{~cm}^{3}, 0.028 \mathrm{~mol}\right), c$ - $\mathrm{HexOH}\left(2.96 \mathrm{~cm}^{3}, 0.028 \mathrm{~mol}^{2}, \mathrm{NEt}_{3}\right.$ $\left(3.9 \mathrm{~cm}^{3}, 0.028 \mathrm{~mol}\right)$ as a white solid, melting point: $95-103^{\circ} \mathrm{C}$, yield $6.841 \mathrm{~g}(49 \%) . R_{f}=0.56\left(\mathrm{CHCl}_{3}: \mathrm{MeOH} 30: 1\right) ;{ }^{1} \mathrm{H} \mathrm{NMR}(500 \mathrm{MHz}$, $\left.\mathrm{CDCl}_{3}\right): \quad \delta=1.22-1.36(\mathrm{~m}, 3 \mathrm{H}) ; 1.46-1.53(\mathrm{~m}, 1 \mathrm{H}) ; 1.57-1.65(\mathrm{~m}, 2 \mathrm{H})$; 1.74-1.78 (m, 2H); 1.87-1.94 (m, 2H); 4.39-4.47 (m, 1H); 7.42-7.47 (m, $4 \mathrm{H}) ; 7.49-7.53(\mathrm{~m}, 2 \mathrm{H}) ; 7.79-7.85(\mathrm{~m}, 4 \mathrm{H}) ;{ }^{13} \mathrm{C} \mathrm{NMR}\left(126 \mathrm{MHz}, \mathrm{CDCl}_{3}\right)$ : $\delta=23.53 ; 25.14 ; 33.88\left(\mathrm{~d}, J_{\mathrm{P}-\mathrm{C}}=3.6 \mathrm{~Hz}\right) ; 75.15\left(\mathrm{~d}, J_{\mathrm{P}-\mathrm{C}}=6.4 \mathrm{~Hz}\right) ; 128.40$ $\left(\mathrm{d}, J_{\mathrm{P}-\mathrm{C}}=13.6 \mathrm{~Hz}\right) ; 131.55\left(\mathrm{~d}, J_{\mathrm{P}-\mathrm{C}}=10.0 \mathrm{~Hz}\right) ; 131.96\left(\mathrm{~d}, J_{\mathrm{P}-\mathrm{C}}=2.7 \mathrm{~Hz}\right)$; $132.25\left(\mathrm{~d}, J_{\mathrm{P}-\mathrm{C}}=138.1 \mathrm{~Hz}\right) ;{ }^{31} \mathrm{P}$ NMR $\left(202 \mathrm{MHz}, \mathrm{CDCl}_{3}\right): \delta=30.49$; GC: 
$\left.t_{\mathrm{R}}=11.03 \mathrm{~min} ; \mathrm{GC}-\mathrm{MS}(\mathrm{EI}, 70 \mathrm{eV}), \mathrm{m} / \mathrm{z}(\%):\left(\mathrm{M}^{+}\right) 300(0.2) ; \%\right) ; 220$ (13); 219 (100); 201 (26); 141 (29). Anal. Calcd for $\mathrm{C}_{18} \mathrm{H}_{21} \mathrm{O}_{2} \mathrm{P}: \mathrm{C} 71.98$, H 7.05. Found: C 72.16, H 7.25.

\section{RESULT AND DISCUSSION}

There are some precedences concerning of the reactivity of arylphosphorus acid amides under Birch reduction conditions [18]. It was decided to check the reactivity of phosphinic acid esters under the same conditions. Therefore, firstly I synthesized a series of diphenylphosphinic acid esters (Scheme 1). Esters (2a-f) were prepared from chlorodiphenylphosphine and appropriate alcohol in the presence of triethylamine, followed by addition of $\mathrm{H}_{2} \mathrm{O}_{2}$. The yields of diphenylphosphinic acid esters varied between $21 \%$ and $81 \%$

Scheme 1

Entry


cd Scheme 1

4

$i$ - $\mathrm{BuOH}$

5

$s-\mathrm{BuOH}$

6

$c-\mathrm{HexOH}$

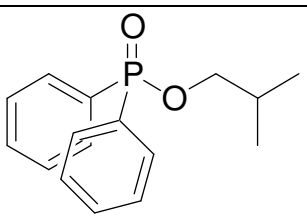

49
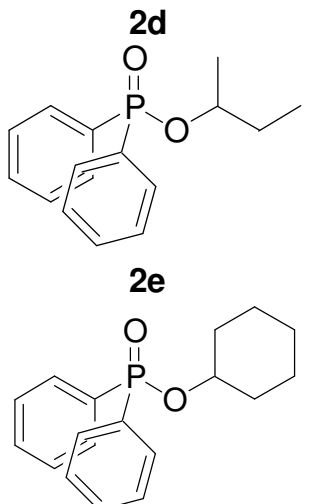

74

81

The methodology used for the synthesis diphenylphosphinic acid esters is known [19], but this paper presents the application of broader spectrum of alcohols leading therefore to a bigger library of phosphorus esters. There are also known other methods of the synthesis of these compounds from $\mathrm{Ph}_{2} \mathrm{P}(\mathrm{O}) \mathrm{Cl}$ with alcohol in the presence of a base [20]. First methodology seems to be better due its flexibility as $\mathrm{H}_{2} \mathrm{O}_{2}$ could be replaced with sulfur [19], nitrogen [22] or $\mathrm{BH}_{3}$-THF [23] leading to different diphenylphosphinic acid esters analogues.

\section{CONCLUSIONS}

I obtained a set of diphenylphosphinic acid esters. These compounds are excellent substrates for nucleophilic substitution reaction which should lead to a huge group of very useful compounds in organic chemistry. Moreover, these structures seem to be a good substrates for Birch reduction reaction. This transformation will provide an access to the 1,4-cyclohexadienyl unit bonded to phosphorus which should a great synthetic potential in the preparation of structurally new organophosphorus compounds. Further application of this methodology is currently under investigation in our laboratory. 


\section{REFERENCES}

[1] T. Imamoto, H. Tsuruta, Chem. Lett., 1996, 707-708

[2] T. Imamoto, T.Oshiki, T. Onozawa, T. Kusumoto, K. Sato, J. Am. Chem. Soc., 1990, 112, 5244-5252

[3] G. Hoge, J. Am. Chem. Soc., 2003, 125, 10219-10227

[4] D. Moulin, S. Bago, Ch. Bauduin, Ch. Darcel, S. Jugé, Tetrahedron: Asymmetry, 2000, 11, 3939-3956

[5] T. Oshiki, T. Imamoto, Bull. Chem. Soc. Jpn., 1990, 63, 3719-3721

[6] U. Nettekoven, M. Widhalm, P. C. J. Kamer, P. W. N. M. van Leeuwen, Tetrahedron: Asymmetry, 1997, 8, 3185-3188

[7] U. Nettekoven, P. C. J. Kamer, P. W. N. M. van Leeuwen, M. Widhalm, A. L. Spek, M. Lutz, J. Org. Chem., 1999, 64, 3996-4004

[8] U. Nettekoven, M. Widhalm, H. Kalchhauser, P. C. J. Kamer, P. W. N. M. van Leeuwen, M. Lutz, A. L. Spek, M. Lutz, J. Org. Chem., 2001, 66, $759-770$

[9] R. Noyori, Tetrahedron, 1994, 50, 4259

[10] H. Brunner, W. Zettlmeier, Handbook of Enantioselective Catalysis, Vol. 2, VCH, Basel, 1993

[11] E. B. Kaloun, R. Merdès, J-P. Genêt, J. Uziel, S. Jugé, J. Organomet. Chem., 1997, 529, 455-463

[12] Ch. Bauduin, D. Moulin, E. B. Kaloun, Ch. Darcel, S, Jugé, J. Org. Chem., 2003, 68, 4293-4301

[13] E. A. Colby, T. F. Jamison, J. Org. Chem., 2003, 68, 156-166

[14] H. Yang, N. Lugan, R. Mathieu, Organometallics, 1997, 16, 2089-2095

[15] R. M. Stoop, A. Mezzetti, Organometallics, 1998, 17, 668-675

[16] F. Maienza, F. Spindler, M. Thommen, B. Pugin, Ch. Malan, A. Mezzetti, J. Org. Chem., 2002, 67, 5239-5249

[17] J. V. Carey, M. D. Barker, J. M. Brown, M. J. H. Russsell, J. Chem. Soc. Perkin Trans I, 1993, 831-389

[18] M. Stankevič, A. Włodarczyk, D. Nieckarz, Eur. J. Org. Chem., 2013, 4351-4371

[19] N. P. Kenny, K. V. Rajendran, D. G. Gilheany, Chem. Commun., 2015, 51, 16561-16564

[20] R. Ramage, B. Atrash, D. Hopton, M. J. Parrott, J. Chem. Soc. Perkin Trans. 1, 1985, 1217-1226

[21] W. Wiegräbe, H. Bock, Chem. Ber., 1968, 101, 1414-1427

[22] T. S. De Vries, S. Majumder, A. M. Sandelin, G. Wang, E. Vedejs, Org. Lett.I, 2012, 14, 688-691 